\title{
The circular loop equation of a cosmic string with time-varying tension in de Sitter spacetimes
}

\author{
Yunqi Liu Hongbo Cheng* \\ Department of Physics, East China University of Science and Technology, \\ Shanghai 200237, China
}

\begin{abstract}
In this work the equation of circular loops of cosmic string possessing time-dependent tension is studied in the de Sitter spacetime. We find that the cosmic string loops with initial radius $r\left(t_{0}\right)>0.707 \mathrm{~L}$, $\mathrm{L}$ de Sitter radius, should not collapse to form a black holes. It is also found that in the case of $r\left(t_{0}\right)<0.707 \mathrm{~L}$ a loop of cosmic string whose tension depends on some power of cosmic time can not become a black hole if the power is lower than a critical value which is associated with the initial size of the loop.
\end{abstract}

PACS number(s): $98.80 . \mathrm{Cq}$

*E-mail address: hbcheng@public4.sta.net.cn 
As a kind of topological defects, cosmic strings including their formation, evolution and observational effects have been a focus for more than twenty years [1-4]. The model was thought as seed of large scale structure in our universe. Although the improved observational data like CMB anisotropy observations and the WMAP experiments do not favour cosmic strings as the origin of the primordial density perturbations giving rise to the existence of galaxies and clusters due to limits on their tension $\left(G \mu \leq 10^{-6}\right)[5-7]$, cosmic strings have several potentially important and distinct astrophysical features. The topological defects, including cosmic strings, were inevitably formed at the end of both conventional inflation [1-3] and brane inflation [8,9], so we have to face and get to know this kind of model. Furthermore cosmic strings still have strong influence on various astrophysics such as gravitational lensing effects [10, 11], gravitational wave background [12, 13], early reionization $[14,15]$ and so on. The model of cosmic string can be used to explain some important astronomical phenomena powerfully. For example, a gravitational lens called CSL-1 invoking two imagines of comparable magnitude of the same giant elliptical galaxy were discovered. It is interesting that many similar objects were found in the vicinity [16, 17].

It is necessary to investigate the evolution of cosmic string loops carefully. Once the cosmic strings formed, they were not static under their own force of tension but envolved instead. Therefore cosmic strings will produce loops unavoidably. The strings can collide and intersect to undergo reconnections. The reconnections of long strings and large loops will generate small loops copiously. In general, string networks consist of long strings and closed string loops. There are observational results which support the existence of cosmic string loops in our Universe [11, 18]. Schild et al. discovered and analyzed the anomalous brightness fluctuations in the multiple-image lens system Q0957+561A, B, which has been studied intensively for 25 years $[11,18]$. The system with brightness fluctuations consists of two quasar images separated by approximately $6{ }^{\prime \prime}$. They thought that the observational results were known to be images of the same quasar not only because of the spectroscopic match, but also because the images fluctuate in brightness, and the time delay between fluctuations is always the same. They suggested that the effect may be due to lensing by an oscillating loop of cosmic string between us and the lensing system, because cosmic string loops supply a quantitative explanation of such synchronous variations in two images. However the fate of cosmic string loops is not entirely satisfactory. According to the past discussions nearly all loops will become black holes except in the special cases. In Minkowski spacetime and Robertson-Walker universe, the loops will collapse to form black holes under their own tension certainly instead of remaining oscillating loops after the loops formation $[19,20]$. In de Sitter backgrounds, only loops with large initial radii can avoid becoming black holes [19, 21, 22]. However a large loop will evolve to be a lot of smaller loops, loops can not live in a de Sitter spacetime unless they are very large. Clearly in the environment with a positive cosmological constant few cosmic string loops can survive. Here we must point out that black holes are final result for loops of cosmic string in our universe bases on the hypothesis that the tension of cosmic string is constant. It may be also worth pointing out that the evolution of loops of different kinds of cosmic strings needs to be 
investigated.

It is really fundamental to explore the evolution of loops of cosmic string with time-dependent tension. In fact the cosmic strings with time-varying tension appeared generally in cosmological situations. So far in nearly all researches, that the tensions of cosmic string are constant is just an assumption. M. Yamaguchi put forward the important issue that the tensions of cosmic strings can depend on the cosmic time [23]. Some researches on the problem were performed and the significant and interesting conclusions were drawn $[23,24]$. The tensions of cosmic strings can depend on the cosmic time. For example, a potential containing one complex scalar field $\phi$ and one real scalar field $\chi$ can be written as $V(\phi, \chi)=\frac{\lambda}{4}\left(|\phi|^{2}-\chi^{2}\right)^{2}+\frac{1}{2} m_{\chi}^{2} \chi^{2}$. The backreaction to the oscillation of field $\chi$ is negligible as the coupling constant $\lambda$ is sufficiently small. The tension of string $\mu$ is associated with the root mean square of the expection value of field $\chi$ and can be denoted as $\mu \propto a^{-3}$ where $a$ is the scale factor which is proportional to $t^{\frac{1}{2}}$ in the radiation-dominated era and $t^{\frac{2}{3}}$ in the matter-dominated era [23]. In the case where the tension depends on the power of the cosmic time like $\mu \propto t^{q}$, such cosmic strings go into the scaling solution when $q<1$ in the radiation domination and $q<\frac{2}{3}$ in the matter domination respectively. The authors of [23, 24] also show that the CMB and matter power spectra induced by cosmic strings with changeable tension can be different significantly from those generated by the conventional cosmic strings with constant tension, which means that a lot of related topics are mysterious and need to be explored. Until now little contribution is made to scrutinize the evolution of cosmic string loops possessing time-dependent tension.

Here we are going to obtain the equation of circular loops of cosmic string with time-varying tension in the de Sitter spacetime due to our accelerating universe. We wonder the time dependence of the tension on the evolution and fate of the cosmic string loops. In the case of constant tension loops in de Sitter backgrounds will keep on expanding if they obey the necessary conditions that their initial radii are larger than $0.707 L, L$ de Sitter radius [21]. Now as the first step we focus on the models with cosmic time power tension. When the tension of cosmic string is proportional to power of cosmic time, we search the new necessary conditions leading the loops to keep enlarging. In this paper first of all we derive the equations of circular loops of cosmic string in the de Sitter spacetime by means of the Nambu-Goto action with an additional factor for the time-dependent tension. We solve the equations numerically to study the evolution of loops and the time dependence of tension on the fate of cosmic string loops, in particular whether the necessary conditions of larger initial size will be revised. The discussions and conclusions are emphasized in the end.

We start to consider the evolution of cosmic string loops whose tensions are functions of cosmic time in a de Sitter spacetime. The metric describing the world is written as,

$$
d s^{2}=\left(1-\frac{r^{2}}{L^{2}}\right) d t^{2}-\frac{d r^{2}}{1-\frac{r^{2}}{L^{2}}}-r^{2}\left(d \theta^{2}+\sin ^{2} \theta d \varphi^{2}\right)
$$

where $L=\sqrt{\frac{3}{\Lambda}}$ is the de Sitter radius associated with the cosmological constant $\Lambda$. A free string propagating in a spacetime sweeps out a world sheet which is a two-dimensional surface. The 
Nambu-Goto action for a cosmic string with time-dependent tension is given by,

$$
S=-\int d^{2} \sigma \mu(t)\left[\left(\frac{\partial x}{\partial \sigma^{0}} \cdot \frac{\partial x}{\partial \sigma^{1}}\right)^{2}-\left(\frac{\partial x}{\partial \sigma^{0}}\right)^{2}\left(\frac{\partial x}{\partial \sigma^{1}}\right)^{2}\right]^{\frac{1}{2}}
$$

where $\mu(t)$ is the string tension and the function of cosmic time. $\sigma^{a}=(t, \varphi)(a=0,1)$ are timelike and spacelike string coordinates respectively. $x^{\mu}(t, \varphi)(\mu, \nu=0,1,2,3)$ are the coordinates of the string world sheet in the spacetime.

For simplicity and without generality we assume that the string lies in the hypersurface $\theta=\frac{\pi}{2}$, then the spacetime coordinates of the world sheet parametrized by $\sigma^{0}=t, \sigma^{1}=\varphi$ can be selected as $x=\left(t, r(t, \varphi), \frac{\pi}{2}, \varphi\right)$.

In the case of planar circular loops, we have $r=r(t)$. According to the metric (1) and the dpacetime coordinates mentioned above, the Nambu-Goto action with an additional factor for the time-dependent tension belonging to a cosmic string denoted as (2) is reduced to,

$$
S=-\int d t d \varphi \mu(t) r\left(h-\frac{\dot{r}^{2}}{h}\right)^{\frac{1}{2}}
$$

giving rise to the following equation of motion for loops which have the time-varying tension for simplicity as $\mu(t)=\mu_{0} t^{q}$,

$$
h^{2} r \ddot{r}+\frac{q}{t} r \dot{r}\left(h^{2}-\dot{r}^{2}\right)+4 h r^{2} \dot{r}^{2}-h \dot{r}^{2}+h^{3}\left(1-2 r^{2}\right)=0
$$

here $h=1-\frac{r^{2}}{L^{2}}$ and $\mu_{0}$ is a constant and we let $L=1$ here. In the case of constant tension as $q=0$, equation (4) is reduced to what has been derived and solved by Larsen [21]. It was shown that loops of cosmic string will keep on expanding in de Sitter spacetimes if their radii satisfy the condition like $r\left(t_{0}\right)>0.707 \mathrm{~L}$ at the moment of their formation, or they will contract to become black holes, which means that the initial radii of expanding loops must be large enough. Now we solve equation (4) numerically. Having performed the burden and surprisingly difficult calculation, we find that our conclusion is the same as Larsen's [21] when the initial radius $r\left(t_{0}\right)>0.707 L$. We discover that in the case of $r\left(t_{0}\right)<0.707 \mathrm{~L}$ there must exist a critical value denoted as $\alpha$. When $q<\alpha$, the cosmic string loops will enlarge to evolve or contrarily will collapse to form black holes when $q>\alpha$. Our findings indicate that the critical value $\alpha$ is negative and associated with the initial size of cosmic string loops, which is depicted in Figure 1. The larger the initial radius $r\left(t_{0}\right)$ is, the smaller the critical value $\alpha$ is. We also show that the cosmic string loops in the case of time-dependent tension with $q>\alpha$ contract faster than ones in the case of constant tension with initial radius of loops like $r\left(t_{0}\right)<0.707 L$ and $q$ is not allowed to be equal to a negative integer unless $\dot{r}\left(t_{0}\right) \neq 0$.

The observational results show that there could exist a lot of cosmic string loops in our universe $[18,25]$. In backgrounds with positive cosmological constant only loops whose radii are larger than a critical value $0.707 \mathrm{~L}$ at the moment of their formations can avoid becoming black holes no matter the strings have constant or changeable tension. In fact the special value $0.707 \mathrm{~L}$ is extremely large 
and can even compares favourably with the size of universe. As we mentioned above the larger loops must evolve to become smaller ones copiously and continuously. The discussions in the case of larger loops of cosmic string exhibit that only fewer loops can survive in our accelerating universe. The conclusion that the only sufficiently large loops of cosmic string remain expanding in the de Sitter spacetime will not let enough cosmic string loops exist. In the case of smaller ones we let the tension is proportional to the power of time denoted as $\mu(t)=\mu_{0} t^{q}$ for simplicity and without losing generality. We find that in de Sitter spacetimes the loops with $q<\alpha$ will expand to evolve instead of becoming black holes. The dependence of negative critical value $\alpha$ on the initial radius of cosmic string loops is shaped in Figure 1. When the power $q$ is negative, the tension will be smaller and smaller and its influence leading the loops contract become weaker and weaker. It is clear that the final results of cosmic string loops will not be black holes if the tensions obey the conditions that the power is lower than a critical value. Certainly the expression of tension can be different, but there must exist the relevant conditions that the expression must satisfy and this kind of cosmic string loops can remain expanding. Our findings indicate that there may be a considerable number of loops of cosmic string in the universe however their tension is time function obeying the necessary conditions, which keeps our observing the effect of cosmic string loops.

The main results of this paper is equation (4), the circular loop equation for a cosmic string evolving in the hypersurface with $\theta=\frac{\pi}{2}$ in the de Sitter spacetime. A larger cosmic string loop with initial radius $r\left(t_{0}\right)>0.707 \mathrm{~L}$ will evolve instead of collapsing to form a black hole no matter whether its tension is constant. The solution to this equation shows that a smaller loop with $r\left(t_{0}\right)<0.707 \mathrm{~L}$ may also never contract to one with a Schwarzschild radius if the expression for tension of cosmic string is a time function satisfying the necessary condition like $\mu(t) \propto t^{q}$ and $q<\alpha$. The critical value $\alpha$ is negative and depends on the initial size of cosmic string loops. The

larger loops need larger absolute value of $\alpha$. Therefore a lot of cosmic string loops can evolve to survive in our accelerating universe.

\section{Acknowledge}

This work is supported by NSFC No. 10333020 and the Shanghai Municipal Science and Technology Commission No. 04dz05905. 


\section{References}

[1] T. W. B. Kibble, Phys. Rep. 67(1980)183

[2] A. Vilenkin, Phys. Rep. 121(1985)263

[3] A. Vielenkin, E. P. S. Shellard, Cosmic Strings and Other Topological Defects, Cambridge University Press 1994.

[4] M. B. Hindmarsh, T. W. B. Kibble, Rep. Prog. Phys. 58(1995)477

[5] L. Pogosian, M. C. Wyman, I. Wasserman, JCAP0409(2004)008

[6] E. Jeong, G. F. Smoot, Astrophys. J. 624(2005)21

[7] M. V. Sazhin et. al., astro-ph/0406516

[8] S. Sarangi, S. H. H. Tye, Phys. Lett. B536(2002)185

[9] L. Pogosian, S. H. H. Tye, I. Wasserman, M. Wyman, Phys. Rev. D68(2003)023506

[10] M. Sazhin et al., Mon. Not. R. Astron. Soc. 343(2003)353

[11] R. Schild et al., Astron. Astrophys. 422(2004)477

[12] T. Damour, A. Vilenkin, Phys. Rev. D71(2005)063510

[13] C. J. Hogan, Phys. Rev. D74(2006)043526

[14] L. Pogosian, A. Vilenkin, Phys. Rev. D70(2004)063523

[15] K. D. Olum, A. Vilenkin, Phys. Rev. D74(2006)063516

[16] M. Sazhin et al., MNRAS 343(2003)353

[17] M. Sazhin et al., Astrophys. J. 636(2005)L5

[18] A. de Laxi, T. Vachaspati, Phys. Rev. D54(1996)4780

[19] M. R. Anderson, The mathematical theory of cosmic string - cosmic strings in the wire approximation, IOP Publishing Ltd., 2003

[20] X. Li, H. Cheng, Class. Quantum Grav. 13(1996)225

[21] A. L. Larsen, Phys. Rev. D50(1994)2623

[22] Z. Gu, H. Cheng, Gen. Rel. Grav. 39(2007)1

[23] M. Yamaguchi, Phys. Rev. D72(2005)043533

[24] K. Ichikawa, T. Takahashi, M. Yamaguchi, Phys. Rev. D74(2006)063526

[25] R. Schild, Astron. J. 129(2005)1225 


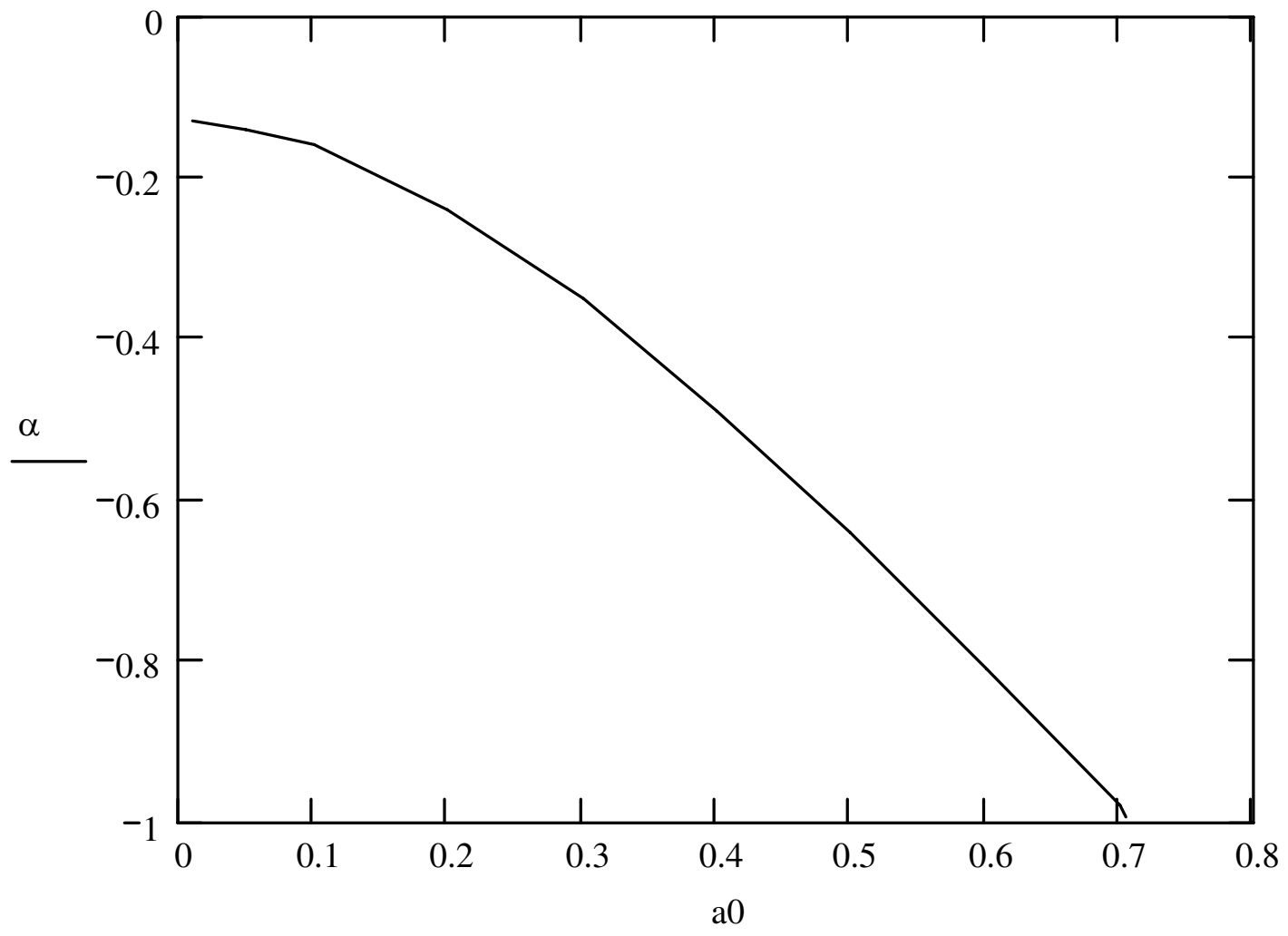

Figure 1: The curve of critical value $\alpha$ as functions of $r\left(t_{0}\right)$ initial radius of circular loops of cosmic string with $r\left(t_{0}\right)<0.707 \mathrm{~L}$ in de Sitter spacetime. 